\title{
Entre circulations individuelles et immobilité familiale : les élites napolitaines face au déclin
}

Thomas Pfirsch

\section{(2) OpenEdition}

\section{Journals}

Édition électronique

URL : https://journals.openedition.org/e-migrinter/289

DOI : 10.4000/e-migrinter.289

ISSN : 1961-9685

Éditeur

UMR 7301 - Migrinter

Édition imprimée

Date de publication : 1 septembre 2013

Pagination : 78-93

ISSN : 1961-9685

\section{Référence électronique}

Thomas Pfirsch, «Entre circulations individuelles et immobilité familiale : les élites napolitaines face au déclin ", e-Migrinter [En ligne], 11 | 2013, mis en ligne le, consulté le 21 septembre 2021. URL http://journals.openedition.org/e-migrinter/289; DOI : https://doi.org/10.4000/e-migrinter.289 


\section{$3^{\text {ème }}$ partie : \\ Circulations et immobilités négociées dans les familles}

\section{Entre circulations individuelles et immobilité familiale : les élites napolitaines face au déclin}

Thomas Pfirsch

$\mathbf{L}$

es travaux sur les nouvelles mobilités des classes supérieures dans le contexte de la mondialisation se sont multipliés ces dernières années. Étudiant les circulations des cadres des firmes multinationales, certains ont formulé l'idée d'une distension des liens entre bourgeoisie et territoire national (Duclos, 2002), voire d'une émergence d'une nouvelle « élite transnationale » (Beaverstock, 2001). D'autres ont au contraire souligné les décalages entre discours et pratiques dans ce milieu des élites de la mondialisation, entre un « imaginaire globalitaire " relevant largement de la posture et des pratiques sociales et patrimoniales encore fortement enracinées localement (Wagner, 1998, 2003 ; Pierre, 2005).
Élites mondialisées et bourgeoisies nationales apparaissent en réalité comme deux milieux étroitement liés, et se caractériseraient moins par leur « déterritorialisation » que par leur capacité à concilier une intense mobilité internationale avec un très fort ancrage local, voire une quasi immobilité résidentielle dans certains beaux quartiers traditionnels (Pinçon et Pinçon-Charlot, 1989). Les classes supérieures constituent donc un milieu particulièrement intéressant pour les études sur les mobilités qui, depuis une vingtaine d'années, tentent de dépasser les oppositions dualistes entre mobilité locale et internationale, migration et sédentarité, circulation et immobilité.

À travers le cas de Naples, cet article étudie les mécanismes par lesquels les classes supérieures parviennent à concilier circulation et ancrage résidentiel local. Il montre en particulier le rôle du patrimoine familial dans cette association. En effet, à Naples et en Italie plus qu'ailleurs, les mobilités individuelles ne peuvent être comprises sans être resituées dans des parcours familiaux et intergénérationnels, la péninsule se caractérisant par des taux très élevés de proximité résidentielle entre membres de la même famille et un rôle décisif de la famille dans l'accès au logement. Cela est encore plus vrai pour les élites de la péninsule, où la localisation du patrimoine immobilier et des entreprises familiales continue à exercer une très forte influence sur les choix résidentiels, contribuant à 
territorialiser durablement des groupes familiaux dans les mêmes quartiers sur plusieurs générations. Les mobilités des individus peuvent être intenses, mais ne prennent sens qu'à l'intérieur de "systèmes résidentiels familiaux» (Lebris et al., 1987 ; Dureau, 2002) ${ }^{1}$ qui, eux, restent très stables voire immobiles d'une génération à l'autre. Pour saisir ces rapports entre circulations individuelles et patrimoine familial, notre étude s'appuie donc sur la collecte d'histoires résidentielles familiales auprès de la vieille bourgeoisie des beaux quartiers du centre de Naples. Des entretiens réalisés en 2005 et 2006 ont permis de reconstituer le parcours résidentiel de cinquante témoins principaux nés dans les années 1940-50², mais aussi ceux de leurs parents et de leurs oncles et tantes, de leurs frères et sœurs, et de leurs enfants adultes, retraçant le parcours géographique de leur parentèle sur trois générations depuis le début du $20^{\mathrm{e}}$ siècle jusqu'à nos jours. Le présent article sera cependant limité à l'étude des fratries des cinquante témoins principaux, soit cent soixante deux individus. L'importance des liens de fratrie dans les phénomènes migratoires a déjà été soulignée (Rosental, 1999), et l'échelle de la fratrie permet d'analyser l'impact d'un même patrimoine familial sur la diversité des mobilités individuelles de frères et de sœurs partageant la même histoire. Qui part? Qui reste? Qui

\footnotetext{
${ }^{1} \mathrm{Au}$ sens d'« ensembles articulés de lieux de résidence des membres d'une famille étendue ou élargie» (Le Bris et al., 1987, p. 258).

${ }^{2}$ Le choix des cinquante individus-témoins s'est fait selon trois critères: l'ancienneté de la fortune familiale (au moins trois générations), la réputation d'appartenir aux «grandes familles » de la ville, et la résidence dans les quartiers aisés du centre. La profession n'a en revanche pas constitué un critère déterminant puisque la position sociale des personnes interrogées repose avant tout sur le patrimoine hérité, et également parce que la polyvalence professionnelle est forte dans les élites italiennes. Mais les individus étudiés appartiennent à trois milieux professionnels principaux: les professions libérales, les entrepreneurs (avec une surreprésentation des constructeurs), et les universitaires. Les cadres d'entreprises sont en revanche nettement sousreprésentés.
}

revient? Les circulations des uns favorisentelles l'immobilité des autres, et inversement?

Ces questions sont essentielles car dans les élites napolitaines, la rétention patrimoniale se double d'une injonction à la mobilité, du fait d'un double mouvement de déclin. Déclin de la ville d'abord, car Naples est passée en cent cinquante ans du statut de grande capitale européenne attractive sur le plan international, à celui de métropole régionale périphérique connaissant une forte émigration de ses élites. Au déclin de la ville s'ajoute celui du milieu particulier des vieilles familles possédantes. Ancienne capitale du Royaume de Naples, la ville avait attiré tout au long du XIX ${ }^{\mathrm{e}}$ siècle les élites terriennes et rentières du Mezzogiorno et elle compte encore aujourd'hui un noyau important de vieilles familles possédantes, nobles ou bourgeoises, au patrimoine considérable. Les fortunes se sont cependant fortement amenuisées depuis 1945 et les vieilles familles ont perdu le contrôle politique et économique de la ville, dont les élites se sont fortement renouvelées (Allum, 1994 ; Brancaccio, Zaccaria, 2003). L'émigration des classes supérieures napolitaines n'est donc pas seulement le fruit des difficultés du marché du travail, elle doit aussi se comprendre - et c'est le cas de beaucoup de migrations d'élites - comme une stratégie de restauration du prestige au sein d'une élite locale en déclin (Hoffmann-Nowotny, 1970 ; Martellini, 1997).

L'étude des parcours résidentiels des cinquante fratries de notre échantillon permet de mieux comprendre ce jeu d'influences réciproques entre rétention patrimoniale et émigration professionnelle, entre circulations individuelles et immobilités familiales. Tout d'abord, elle montre la force d'un modèle résidentiel local et patrimonial dans les vieilles familles napolitaines. La majorité des parcours résidentiels prend la forme d'un maintien à Naples, qui peut s'accompagner d'une mobilité assez intense mais spatialement circonscrite au sein d'un espace patrimonial 
stable (I). Cette rétention patrimoniale est cependant compatible avec une circulation nationale et internationale, les individus étant ramenés à Naples par les entreprises familiales, les mises à disposition de logements prestigieux ou les maisons de villégiature (II). Rétention patrimoniale et émigration professionnelle sont en effet interdépendantes chez les élites de la ville pour lesquelles elles font véritablement système (III).

\section{Dans les élites napolitaines : la force d'un modèle résidentiel local et patrimonial}

\section{Une très forte proximité familiale, typique des pays d'Europe du sud}

Les cinquante familles de notre échantillon se rattachent au modèle résidentiel sud-européen de proximité familiale (Höllinger, Haller, 1990; Allen et al., 2004 ; Pfirsch, 2011). Les parentèles y apparaissent soudées et très concentrées dans l'espace: parents et enfants adultes, frères et sœurs, oncles et neveux résidant bien souvent dans le même quartier, la même rue ou dans des appartements indépendants du même immeuble. Ainsi, au moment de l'entretien, trente-quatre des cinquante témoins principaux résidaient dans le même quartier qu'un membre de leur parentèle, et dix-neuf d'entre eux (38\%) habitaient dans un immeuble où vivaient également des parents proches, à d'autres étages ou sur le même palier.

Cette très forte proximité familiale, dans un milieu aisé qui pourrait a priori se passer du soutien de la parentèle dans la vie quotidienne ne doit pas étonner. ${ }^{3}$ Si en Europe du nord la proximité résidentielle familiale est plus élevée dans les classes populaires, où elle sert à gérer le quotidien (Bonvalet, Maison, 1999: 35), en Italie et

${ }^{3}$ Dans les familles étudiées, l'aide domestique quotidienne est assurée par du personnel salarié et non par des proches. dans les autres pays d'Europe du sud (Grèce, Espagne, Portugal) elle renvoie plutôt à des facteurs culturels et est donc répandue dans tous les milieux sociaux. Dans ces pays, la proximité familiale est moins liée à la nécessité de l'entraide familiale qu'à un attachement plus général à un "style de vie fondé sur les interactions constantes entre membres de la parentèle» (Barbagli et al., 2003: 203), caractéristique des systèmes familiaux à liens forts (Höllinger, Haller, 1990). Elle est aussi la conséquence d'un modèle de formation de la famille fondé sur l'installation des enfants par les parents au moment du mariage dans un logement en propriété (Barbagli et al., 2003). Dans ces pays de propriétaires que sont devenus l'Italie, la Grèce ou l'Espagne - et où le mariage demeure essentiel dans la formation de la famille - les jeunes adultes sont en quelque sorte "installés » par leurs parents qui leur mettent à disposition un logement au moment de leur mariage, et le font en général à proximité de chez eux. La force de ces modèles culturels se retrouve clairement dans les familles étudiées. Les deux tiers des personnes interrogées voyaient ainsi un membre de leur parentèle proche tous les jours ${ }^{4}$. Pour la majorité d'entre eux, la proximité familiale est clairement valorisée dans les discours. Enfin et surtout, la grande majorité des cinquante individus témoins a été retenue à proximité de chez leurs parents par des mises à disposition de logements de famille à l'occasion de leur installation en couple.

\section{Un modèle résidentiel patrimonial : la reprise des logements de famille par les enfants mariés}

En effet, la spécificité de notre échantillon réside dans le fait qu'ici, la mise en œuvre des modèles culturels sudeuropéens a été facilitée par une forte emprise patrimoniale sur l'immobilier de la ville. L'installation des enfants par les parents se fonde autant sur des achats de

${ }^{4}$ Il s'agit bien sûr de membres de leur parentèle ne résidant pas avec les enquêtés. 
logements que sur la transmission de logements de famille. Ainsi, sur les cinquante personnes interrogées, plus de la moitié (vingt-huit) ont obtenu leur logement actuel par donation, héritage ou mise à disposition gratuite par un de leurs ascendants, principalement leurs parents, et en général à l'occasion de leur mariage. C'est ce système de reprise des logements de famille par les enfants qui explique les taux extrêmement élevés de proximité familiale dans les familles étudiées - et en particulier le poids des regroupements familiaux dans les mêmes immeubles. Il y a dans la bourgeoisie napolitaine un véritable modèle résidentiel patrimonial fondé sur la reprise des logements de famille par les enfants qui contribue à maintenir des familles entières dans les mêmes rues et les mêmes immeubles sur plusieurs générations.

L'ampleur des patrimoines immobiliers dans les familles étudiées s'explique par le rôle déterminant de la spéculation immobilière dans les classes supérieures de l'Italie méridionale. Avec la crise de la rente foncière dans les années 1876-1900, la bourgeoisie napolitaine s'est largement reconvertie dans la rente urbaine qui a continué à jouer un rôle essentiel pour les élites locales tout au long de la période de forte croissance de la ville jusqu'à la fin des années 1970, devenant l'une des bases principales de l'économie locale et l'un des piliers du système politique de la ville (Allum, 1994). Entrepreneurs, médecins, avocats se sont alors constitués de vastes patrimoines immobiliers dans les beaux quartiers de l'ouest de Naples (Chiaia, Posillipo, Vomero), composés d'immeubles de rapport, mais aussi d'immeubles familiaux destinés à loger les membres de leur parentèle. Les immeubles de famille sont ainsi encore fréquents dans les beaux quartiers. Aujourd'hui très divisés, vendus par étages ou par vastes unités de surface, ils continuent souvent à abriter des descendants du propriétaire initial et ont été largement utilisés pour mettre à disposition des logements prestigieux aux enfants mariés.

\section{Une rétention familiale source d'immobilité résidentielle?}

Ce modèle patrimonial exerce donc une forte rétention sur les parcours résidentiels. Le type de parcours majoritaire dans notre échantillon est celui du maintien à Naples: sur les cent soixante-deux membres des cinquante fratries étudiées, soixante-cinq individus n'ont jamais quitté la ville au cours de leur itinéraire résidentiel et un peu plus de la moitié d'entre eux n'ont même jamais quitté les beaux quartiers...

Ces individus retenus à Naples présentent deux grandes caractéristiques communes. Sur le plan professionnel, il s'agit essentiellement d'indépendants (chefs d'entreprise - en particulier dans le secteur de la construction - et membres de professions libérales), et dans une moindre mesure de salariés du public (professeurs d'université notamment), alors que les cadres du privé sont sous-représentés. Le maintien à Naples a donc ici clairement été facilité par la présence d'entreprises ou de clientèles familiales, dans des milieux marqués traditionnellement par une forte reproduction professionnelle. Sur le plan familial, il s'agit souvent d'individus occupant les derniers rangs des fratries: pour eux, la réinstallation à Naples a été facilitée car elle coïncidait à quelques années près avec la succession des grands-parents, ce qui a permis une mise à disposition plus rapide de logements de famille prestigieux. Ces parcours de maintien à Naples s'inscrivent donc dans la plupart des cas dans un processus de rétention familiale. À la rétention des liens familiaux s'est ajoutée ici une très forte rétention patrimoniale, par le biais des entreprises familiales comme par celui des logements de famille.

Mais cette rétention familiale est-elle pour autant source d'immobilité résidentielle? Pas toujours. Le système de mise à disposition des logements de famille peut donner lieu à une assez forte mobilité, mais à très petite distance, qui prend la 
forme d'une circulation familiale : la majorité des logements occupés par un individu au cours de sa vie sont en fait des logements de famille, si bien que ce dernier circule à l'intérieur d'un espace connu et circonscrit, limité aux beaux quartiers de la ville. Le cas de Federico, un architecte né en 1947 et issu d'une vieille famille noble en fournit un bon exemple. Ce dernier a passé toute son enfance dans la villa de sa famille maternelle, une riche famille d'industriels, aux côtés de son grand-père, d'un oncle et d'une tante, qui habitaient chacun dans des appartements indépendants dans la villa. Mais à la mort de son grand-père maternel, en 1971, Federico a déménagé dans une autre maison de famille, paternelle cette fois : un grand palais nobiliaire où vivaient également des oncles et des cousins. Il y a vécu un an avec sa mère au deuxième étage avant d'emménager tout seul à l'étage supérieur. Puis, en 1979 il a quitté le palais familial pour aller habiter plus loin dans la même rue. Sept ans plus tard, Federico est cependant revenu dans le palais paternel, dans un appartement beaucoup plus grand situé au $3^{\mathrm{e}}$ étage et acheté avec l'argent de la revente du précédent. Enfin, en 2005, Federico a de nouveau déménagé dans le palais familial en passant du $3^{\text {e }}$ au $5^{\mathrm{e}}$ étage, finissant par s'installer dans l'appartement où son père avait passé son enfance. Au total, il aura donc changé six fois de logement au cours de sa vie, mais cinq de ces six logements sont en fait des logements de famille et quatre d'entre eux se situent d'ailleurs dans le même palais familial. Voici donc une personne qui a passé quasiment toute sa vie dans des logements de famille, mettant largement à profit pour se loger le vaste patrimoine immobilier de ses deux familles d'origine, si bien que malgré des déménagements nombreux, sa trajectoire géographique est en fait extrêmement simplifiée, se réduisant pour l'essentiel à un passage de la villa maternelle au palais paternel, dans un rayon de moins de cinq kilomètres au sein des quartiers aisés de Naples (figure 1). On voit ici que la rétention patrimoniale ne provoque pas forcément une immobilité résidentielle, mais plutôt une «micro-mobilité », pour reprendre l'expression des démographes historiques, à savoir des changements de logements nombreux mais très circonscrits spatialement et "parfaitement compatibles avec le modèle de sédentarité » (Poussou, $1988: 20)$.

En revanche, à l'échelle intergénérationnelle, la rétention familiale est clairement source d'immobilité, au sens où elle stabilise durablement une famille dans un immeuble, une rue ou un quartier. En effet, ces trajectoires de circulation familiale locale sont souvent répétitives. Le rôle des logements de famille dans la structuration des trajectoires résidentielles aboutit à l'existence de parcours types dans certaines familles, la même trajectoire résidentielle pouvant se retrouver chez des frères et des sœurs, ou chez des parents et leurs enfants à une génération d'écart. La famille paternelle de Giuseppe, un psychanalyste né en 1947, en fournit un bon exemple. Son arrièregrand-père avait en effet acheté à la fin du XIX $^{\mathrm{e}}$ siècle deux grands immeubles familiaux: un vieux palais du centre historique où il a vécu avec sa famille, et un grand immeuble bourgeois des beaux quartiers où se sont réinstallés progressivement sept de ses neuf enfants après sa mort en 1920. Ces deux immeubles familiaux ont alors structuré la plupart des trajectoires des descendants de cet arrièregrand-père pendant près de trois générations, le passage de l'immeuble du centre historique à celui des beaux quartiers constituant un véritable paradigme familial que l'on retrouve chez cinq individus de la lignée paternelle de Giuseppe: son grandpère paternel, une grande-tante paternelle, son père, et deux tantes paternelles. Ainsi, à Naples, le grand-père paternel et le père de Giuseppe ont habité les mêmes appartements, dans le même ordre, au cours de leur vie. La famille de Giuseppe est 
encore aujourd'hui majoritaire dans la copropriété de l'immeuble. On est donc ici dans un système résidentiel familial au sens plein du terme, car pour qu'il y ait système il faut «que la mobilité interne existe», la «mobilité apparente du logement» s'opposant à la «stabilité du système résidentiel » (Lebris et al., 1987 : 258).

Figure $\mathbf{n}^{\circ} 1$ : le parcours de Federico : une circulation familiale locale.

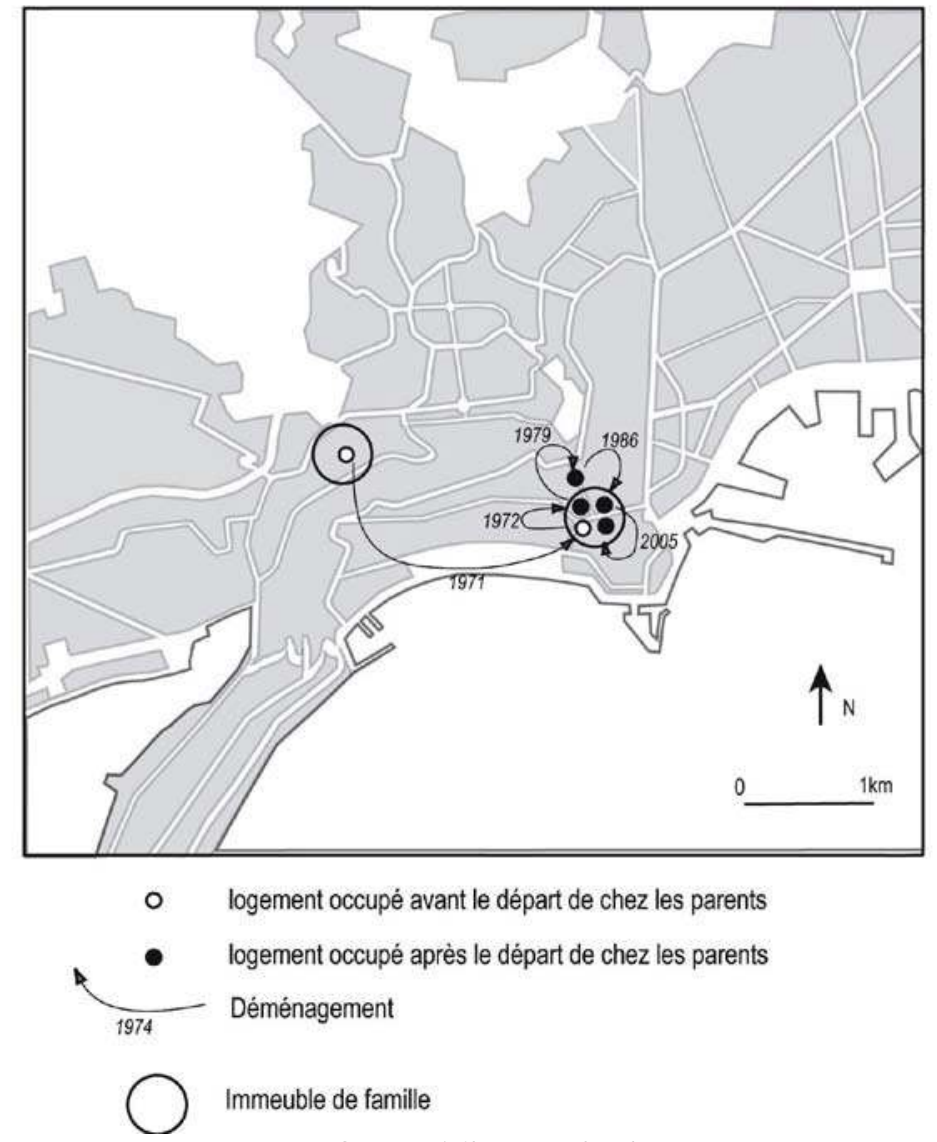

Source: Thomas Pfirsch.

Un modèle résidentiel de plus en plus difficile à reproduire : le déclin des grandes familles

Depuis les années 1970, période à partir de laquelle les cinquante individus témoins ont commencé à quitter le logement de leurs parents, ce modèle résidentiel familial et patrimonial est de plus en plus difficile à satisfaire, du fait d'un double déclin. Au morcellement des patrimoines, prononcé dans les familles dont la fortune remonte à plus de trois générations, s'est ajouté le déclin économique de la ville, très fort depuis la fin de la politique d'intervention de l'État italien en faveur du Mezzogiorno au cours des années 1980. La trajectoire des entreprises familiales de notre échantillon confirme la fragilité des élites économiques napolitaines, très dépendantes de la commande publique et constamment renouvelées en fonction du système politicoclientéliste local (Brancaccio, Zaccaria, 2003). Beaucoup d'entreprises, en particulier dans le secteur de la construction, n'ont pas résisté aux crises politiques de la Libération, puis à celles du début des années 1990 (scandale tangentopoli), contraignant une part importante des individus étudiés à se tourner vers le salariat - encore rare à la génération de leurs parents -, ce qui les rend beaucoup plus sensibles aux difficultés du marché du travail local et à l'attractivité des métropoles européennes. 


\section{Migrer face au déclin : l'importance des circulations nationales et internationales dans les élites napolitaines}

\section{Une noria migratoire entre Naples et les métropoles européennes}

La rétention patrimoniale s'accompagne en effet d'une intense circulation nationale et internationale dans les familles étudiées. Ainsi, sur les cent soixante-deux membres des fratries étudiées, quatre-vingt-huit individus (donc plus de la moitié) ont quitté Naples définitivement ou temporairement au cours de leur parcours résidentiel adulte pour s'installer ailleurs, en Italie ou à l'étranger. Cette émigration des élites napolitaines, interne ou internationale, ne cadre que partiellement avec les caractéristiques des grandes migrations de travail italiennes d'origine populaire. Sur le plan des destinations, l'émigration des élites napolitaines associe une migration interne sud-nord - largement majoritaire puisque seuls vingt-sept enquêtés sont passés par l'étranger - et une migration internationale, comme c'est le cas pour les migrations ouvrières des années 1945-1975. Mais à une échelle plus locale, des différences nettes apparaissent (figure 2). En ce qui concerne l'étranger, le nouveau monde est peu représenté et l'Europe domine nettement, en particulier les capitales d'Europe du nordouest (Londres, Paris, Bruxelles). En Italie, les destinations sont encore plus déséquilibrées avec une très nette domination des deux capitales de la péninsule, Milan et surtout Rome (villes par lesquelles sont passés vingt des enquêtés au cours de leur parcours résidentiel). Au total, ces destinations métropolitaines reflètent bien la nature d'une émigration qualifiée, composée de cadres d'entreprise et de hauts fonctionnaires, et orientée vers les centres de commandement italiens ou européens plus que vers les villes industrielles et les destinations traditionnelles de l'émigration italienne de masse. Le poids de Rome s'explique par sa proximité avec Naples et par des logiques gravitaires, mais il renvoie également au profil professionnel des élites napolitaines, où dominent les professions intellectuelles salariées, les hauts fonctionnaires et les cadres de grandes entreprises publiques ayant leur siège à Rome. La romanisation croissante des familles de la bourgeoisie napolitaine est le reflet de la dépendance de l'économie de la ville vis-à-vis des centres de décision de la capitale.

Mais qu'elle soit interne ou internationale, cette émigration des élites napolitaines est majoritairement restée provisoire. Les parcours résidentiels montrent l'importance des retours, qui ont concerné cinquante-six individus, soit près des deux tiers des quatre-vingt huit émigrés enquêtés, et $35 \%$ du total de la population étudiée. Au moment de l'entretien, douze personnes avaient même choisi de s'installer dans un système de bi-résidence entre Naples et Rome ou Naples et Milan. Ainsi, plus que l'émigration proprement dite, c'est l'importance des circulations entre Naples et les grandes métropoles italiennes et européennes qui caractérise notre échantillon, en particulier entre Naples et Rome. Une noria professionnelle s'est mise en place au sein des élites napolitaines au cours de l'entre-deux guerres, le passage par une entreprise milanaise ou une administration romaine devenant de plus en plus fréquent dans les parcours résidentiels. Comment comprendre cette importance des départs, puis celle des retours à Naples ? 


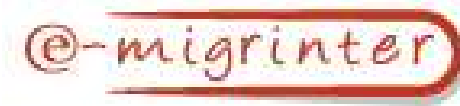

Figure $\mathrm{n}^{\circ} 2$ : Les destinations de l'émigration des élites napolitaines.

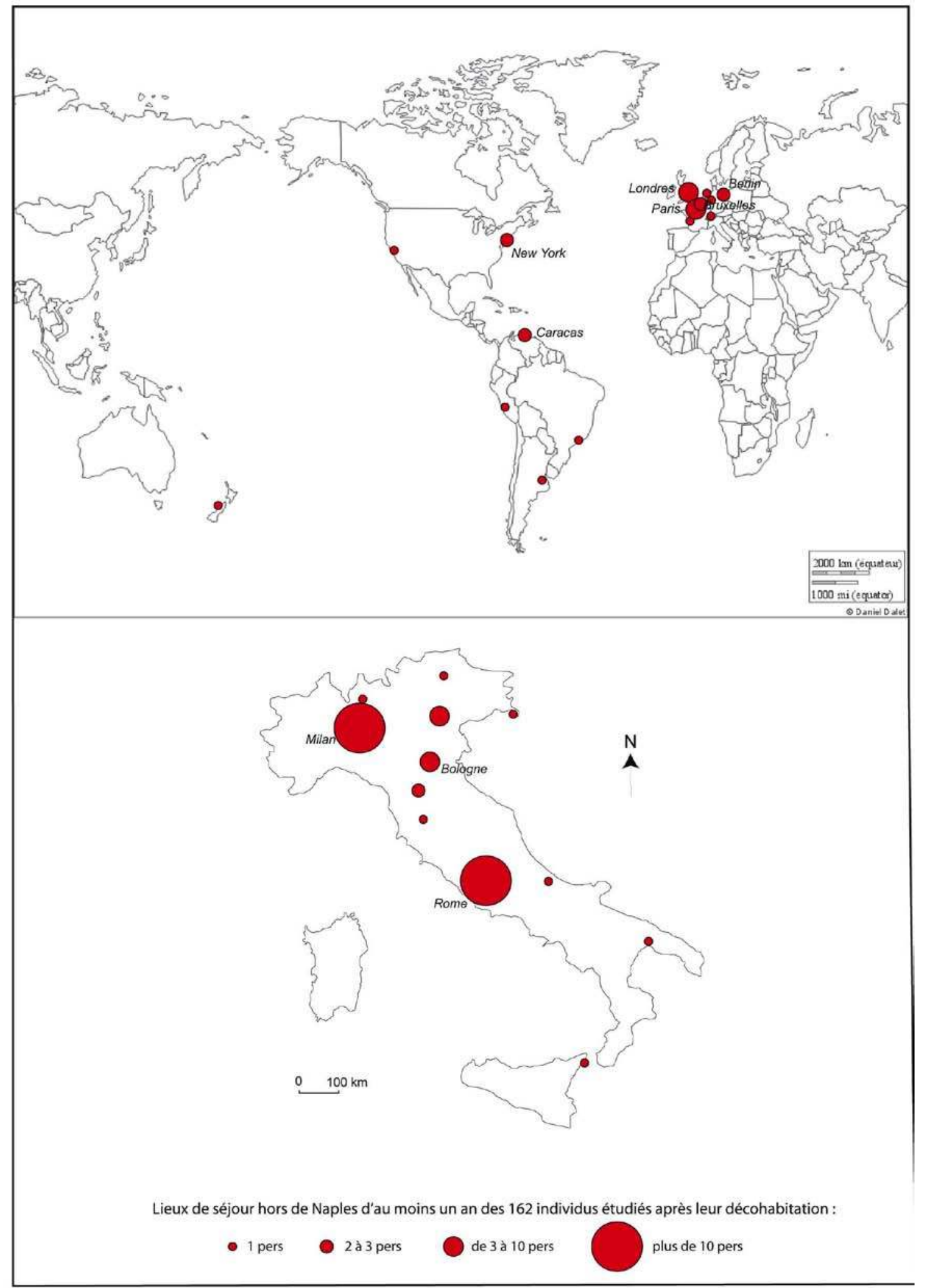

Source: Thomas Pfirsch. 


\section{Cadres salariés, fratries nombreuses, aînés déchus : ceux qui partent}

Les enquêtés qui ont quitté Naples pour Rome, l'Italie du Nord ou l'étranger ont trois caractéristiques communes. Tout d'abord, il s'agit principalement de personnes ayant quitté Naples alors qu'ils étaient jeunes adultes: hommes en début de carrière ou femmes suivant leur mari. En revanche, la quasi-totalité d'entre eux a fait ses études à Naples, ville qui peine à retenir ses élites mais qui continue à bien les former, forte d'une université ancienne et prestigieuse. D'autre part, sur le plan socio-professionnel, la grande majorité de ces émigrés sont des salariés - cadres d'entreprise ou fonctionnaires - alors que ces derniers sont nettement sous-représentés dans notre échantillon où dominent les professions libérales et les entrepreneurs. Il s'agit donc des membres des familles étudiées qui n'ont pas forcément pu s'appuyer sur une entreprise familiale et ont été confrontés aux difficultés du marché du travail napolitain.

Enfin, sur le plan familial, les émigrés de notre échantillon appartiennent à des fratries nombreuses $(3,4$ personnes en moyenne) et se situent souvent dans les premiers rangs des fratries. Ici, le modèle de la réinstallation des enfants adultes dans des logements de famille a en effet été compliqué par le nombre des enfants, en imposant un morcellement fort $\mathrm{du}$ patrimoine et l'émigration définitive ou provisoire de certains membres de la fratrie, en particulier les premiers nés, dont la décohabitation a souvent eu lieu avant la mort et la succession des grands-parents, et pour lesquels les mises à disposition de logements de famille interviennent donc plus tard dans le cycle de vie. Outre ce retard de la transmission patrimoniale, les aînés sont aussi les premiers porteurs du prestige familial, ce qui fait peser sur eux une pression sociale plus forte et peut les inciter à partir. Les familles étudiées fournissent en effet de nombreux exemples d'ainés élevés dans la richesse et supportant mal un déclin économique ne correspondant plus à un prestige familial dont ils s'estiment porteurs. C'est le cas de Francesco A. né en 1940 et ainé d'une fratrie de trois. Alors que sa sœur et son frère cadets se réinstalleront dans des appartements du palais familial napolitain et ne quitteront jamais la ville, lui essaye sans succès de relancer l'usine paternelle de Salerne et part ensuite dans les années 1960 essayer de refaire fortune au Vénézuéla. C'est également le cas de Pasquale P., né en 1943 et lui aussi aîné d'une fratrie de trois, descendant d'une grande famille noble napolitaine par son père et par sa mère d'une riche famille d'industriels italiens ayant fait fortune au Brésil. Alors que son frère, architecte, et sa sœur, musicienne, restent à Naples, lui part pendant sept ans au Brésil, dans les années 1970, tenter de travailler dans la société familiale désormais contrôlée par une autre branche de la lignée, avant de revenir s'installer dans la maison de famille des environs de la ville où il vit des locations de ses appartements napolitains.

Ainsi l'émigration élitaire napolitaine ne renvoie pas seulement à la crise du marché du travail local. Elle est aussi liée à des dynamiques sociales de décalage entre prestige et pouvoir et peut s'interpréter dans le cadre des théories insistant sur le rôle des groupes de référence dans la formation des projets migratoires (Hoffmann-Nowotny, 1970 ; Rosental, 1999). Les individus qui quittent Naples ont tendance à le faire lorsque leur pouvoir social réel devient nettement inférieur à leur prestige au sein du groupe de référence que constituent les vieilles familles de la bourgeoisie des beaux quartiers de la ville, qui forment un réseau dense où réseaux de parenté et réseaux amicaux s'entrecroisent (Pfirsch, 2008). Rester à Naples impliquerait de se confronter à «un univers social perdu», tandis que «le départ constitue l'une des manières possibles de réagir à une comparaison devenue écrasante » avec les autres grandes familles (Rosental, 1999: 192). Mais comment, dès lors, comprendre l'importance des retours, alors même que la 
situation du marché de l'emploi de la ville s'est fortement dégradée depuis la fin des années 1970 ? Et comment comprendre la différence de choix entre ceux qui sont partis définitivement et ceux qui ont décidé de rentrer à Naples?

\section{Retours et « rappels familiaux »}

Au sein des cinquante fratries étudiées, le profil des émigrés définitifs et des émigrés provisoires est très proche sur le plan professionnel. En revanche, leurs situations familiales sont différentes. Le rôle du conjoint apparait déterminant dans les choix de retour. La grande majorité des émigrés rentrés à Naples avait un fiancé ou un conjoint napolitain avant de partir à Rome ou à l'étranger (83\%), alors que ceux qui ne sont pas rentrés ont majoritairement épousé des conjoints non napolitains. Les entretiens offrent de nombreux exemples de personnes ramenées à Naples par leur conjoint, en particulier par leur femme (dont le rôle dans l'animation de la sociabilité familiale est très fort, ici comme ailleurs en Italie), et au contraire de personnes peu à peu attirées dans le réseau social de leur conjoint originaire du nord de la péninsule. C'est donc souvent par un processus de « rappel familial» (Bertaux-Wiame, 1991) que les émigrés sont rentrés à Naples. Ce rappel peut s'exercer par le biais du conjoint, mais il est aussi lié à la concentration résidentielle de ces familles fortes napolitaines. En effet, la présence d'un immeuble de famille où vivent plusieurs ménages apparentés crée ce que les anthropologues ont appelé un kin nodule (Firth et al., 1969), un lieu de concentration des contacts où l'information et la mémoire familiales s'entretiennent, ce qui contribue à polariser les mobilités de toute la parentèle et à maintenir sa cohésion.

Mais, dans notre échantillon, le rappel relationnel dû à la force des liens familiaux se double souvent d'un rappel patrimonial, le retour à Naples coïncidant avec une donation, une succession ou la mise à disposition gratuite d'un logement de famille aux émigrés. La période passée en dehors de Naples a en effet laissé le temps aux familles d'origine de préparer le retour, en réorganisant le parc de logements de famille pour pouvoir reloger le ménage émigré dans un appartement prestigieux des beaux quartiers de la ville. Ce processus donne lieu à des parcours résidentiels en forme de boucles: les individus, après une période passée à Rome ou à l'étranger, rentrent s'installer dans les quartiers, les rues, voire les immeubles où ils ont passé leur enfance, dans un logement ayant appartenu à leurs parents ou leurs grands-parents. Sur les cent soixante-deux membres des fratries étudiées, quarante-six individus ont connu une telle trajectoire de boucle familiale. C'est le cas de Giuseppe psychanalyste né à Naples en 1947, issu de familles de riches entrepreneurs. Ses deux familles d'origine, maternelle et paternelle, possédaient chacune un grand immeuble de famille sur une avenue prestigieuse des beaux quartiers de Naples. Pourtant, lors de son départ du foyer parental en 1975, Giuseppe n'a pas reçu de logement dans les immeubles familiaux comme cela avait été le cas pour son père ou son grand-père. La fortune de la famille s'était en effet considérablement amoindrie depuis la Deuxième Guerre mondiale. De plus, Giuseppe n'a pas suivi les modèles résidentiels traditionnels de sa famille et de son milieu. Le départ de chez ses parents a été préparé par de longues études universitaires entre Pise, Urbino et Naples. Puis, lorsqu'il est revenu dans la ville après deux ans passés à Milan et Londres (en suivant les traces de son frère aîné et de son père, passés également par Milan une génération auparavant), Giuseppe s'est installé quelques années dans un quartier populaire du centre de Naples. Pourtant, en 1984, à la faveur de la succession de son grand-père, il est retourné dans la «città bene» (les beaux quartiers) et a emménagé dans l'immeuble de sa famille maternelle en même temps que sa mère et sa sœur. Après une trajectoire complexe et une mobilité élevée qui l'a porté à Milan, Pise ou Londres, Giuseppe est donc revenu s'installer dans la 
rue où il a passé son enfance, dans l'appartement où a vécu son grand-père maternel et dans un immeuble où vivaient également sa sœur, des oncles et des cousins.

Enfin, même lorsque l'émigration est définitive, le rappel familial peut s'exercer par le biais des maisons de famille des environs de Naples, la circulation résidentielle s'accompagnant souvent d'une grande stabilité des pratiques de villégiature. La famille d'Alessandra T., une architecte née à Naples en 1938, en fournit un bon exemple. Cette fratrie nombreuse est issue d'une famille d'armateurs reconvertie dans le salariat et les professions libérales. Sur les huit membres de la fratrie, trois ont quitté Naples définitivement dans les années 1960 et 1970 : un frère aîné et une sœur cadette d'Alessandra sont partis à Rome, où ils ont fait carrière dans deux grandes entreprises publiques, tandis qu'un de ses frères a travaillé comme haut fonctionnaire à Bruxelles, avant de s'établir à Berlin. Ceux qui sont restés ne travaillent pas dans le monde de l'entreprise et sont soit universitaires, soit membres de professions libérales. Malgré cette forte mobilité internationale, la famille a maintenu des liens forts avec Naples. Ceux-ci reposent d'abord sur la conservation d'un kin nodule dans les beaux quartiers de Naples. Dispersée à l'échelle nationale et européenne, la famille d'Alessandra est en effet concentrée dans l'espace de la ville (figure 3): Alessandra et son frère aîné vivent dans la maison de famille construite par leur grand-père paternel qui a été divisée en deux appartements, tandis que la mère d'Alessandra habite avec une de ses filles dans une rue voisine du même quartier. Ce « noyau de parenté » réunissant une mère et trois de ses enfants adultes dans un rayon de moins d'un kilomètre multiplie donc les occasions de contacts et de retours pour les membres de la famille ayant quitté la ville. Mais c'est surtout grâce à la conservation de la grande maison de famille de Castellammare di Stabia, dans le golfe de Naples, lieu de l'enfance du grand-père paternel et berceau de la famille que cette parentèle dispersée maintient des liens forts avec Naples. Alessandra et trois de ses frères et sœurs possèdent encore des appartements dans cette immense villa où toute la famille se retrouve tous les mois d'août, ceux de Naples comme ceux de Rome, autour d'un tournoi de football familial dans le jardin ou d'un concert de l'orchestre de famille.

On le voit, dans les familles étudiées l'émigration ne remet pas nécessairement en cause l'ancrage local à Naples. Le passage par Milan, Londres ou Rome apparaît même nécessaire à la reproduction du modèle résidentiel patrimonial, la noria migratoire libérant temporairement la pression sur le parc de logements de la famille et permettant au final à la majorité des membres d'une fratrie de se réinstaller dans les rues les plus sélectes de la ville. 


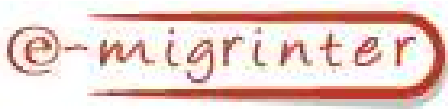

Figure $\mathbf{n}^{\circ} \mathbf{3}$ : Une famille entre dispersion nationale et concentration locale.

La parentèle proche d'Alessandra à Naples (2005)

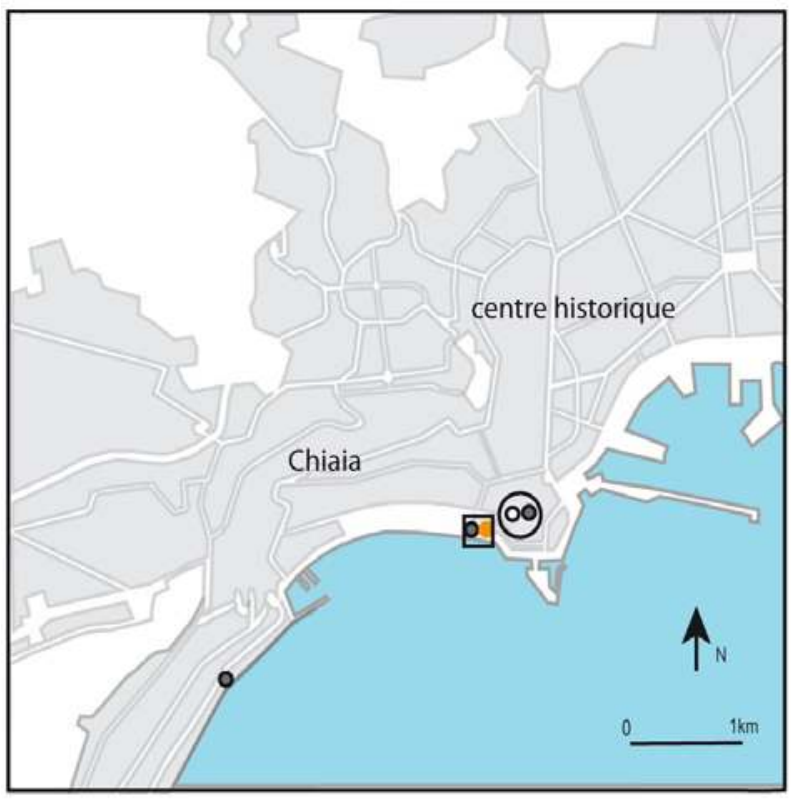

La parentèle proche d'Alessandra en Italie (2005)

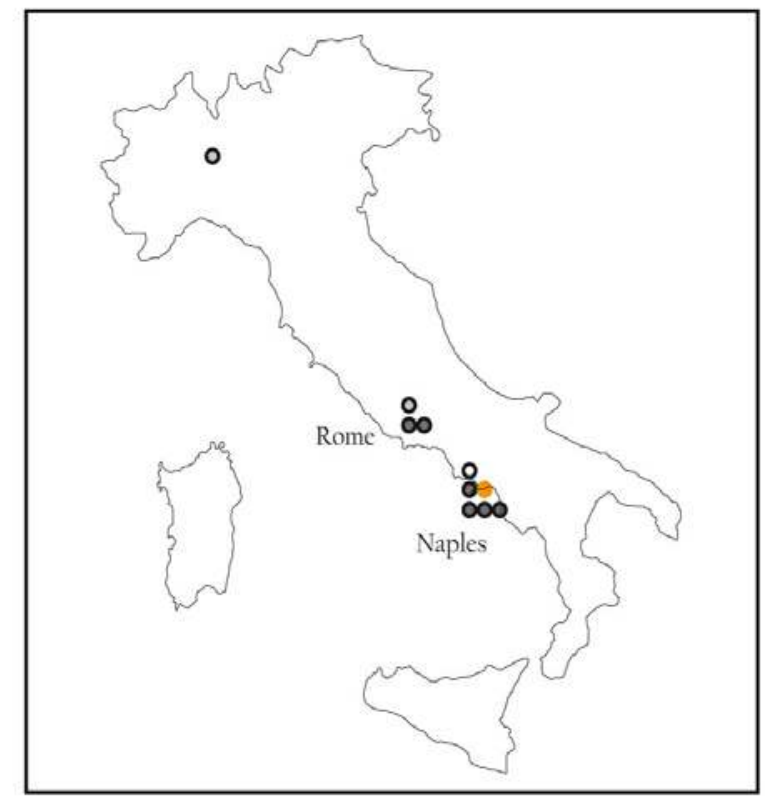

Lieux de résidence :

- d'ego et éventuellement de son conjoint
- des enfants d'ego ayant décohabité
- des frères et soeurs d'ego
durents d'ego
duère d'ego (si seul)
- de ses grands-parents paternels
de ses oncles et tantes paternels

- de ses oncles et tantes maternels

de la mère d'ego (si seule)

de ses grands-parents matemels

- de ses beaux-parents

- de ses cousins germains paternels

- de ses cousins germains maternels

- de ses neveux

Source : Thomas Pfirsch.

\section{Quand les circulations permettent l'immobilité : le fonctionnement des systèmes résidentiels familiaux}

Dans ces vieilles familles en déclin, circulation professionnelle et rétention patrimoniale ne s'opposent pas, elles font au contraire système. Le modèle de réinstallation des enfants mariés dans un logement de famille n'est en effet pas toujours aisé à réaliser et demande du temps, celui des détails de succession, des négociations familiales, des travaux de division des appartements. Pour fonctionner, le système a besoin d'une circulation des membres de la famille pour permettre d'ajuster régulièrement le parc de logements aux besoins de la parentèle. Il a besoin d'expulser pour pouvoir retenir, et les entretiens permettent de décrire les stratégies mises en place par les familles napolitaines pour organiser à la fois la circulation des uns et la réinstallation des autres.

\section{Retard des mises à disposition de logements et boucles familiales}

Les entretiens montrent que la reprise des logements de famille intervient relativement tardivement, en général plusieurs années après le mariage. La division de l'appartement parental en logements indépendants implique de lourds travaux, tandis que la reprise d'un appartement à la suite d'une succession intervient nécessairement assez tard dans le cycle de vie, après la mort des grandsparents, et doit de toute façon attendre que les démarches juridiques de la succession soient terminées. C'est donc souvent de cinq à dix ans, et parfois plus, qu'il faut attendre avant de pouvoir s'installer véritablement dans un logement en propriété à proximité 
de chez les parents. Même lorsqu'ils restent à Naples, les enfants qui reprennent des logements de famille après leur mariage le font donc en général au terme d'une trajectoire en boucle. Ils quittent quelques années les beaux quartiers de la ville pour aller louer un appartement dans un quartier voisin, souvent avec l'aide de leur famille, en attendant que le logement de famille et qui leur est destiné soit disponible. Le cas de Patrizzia, une enseignante et fille de médecin née en 1949 le montre bien. Son père lui avait acheté un appartement à l'étage d'en dessous de chez lui, dans le même immeuble, en pensant à son mariage mais lorsque Patrizzia s'est mariée en 1975, l'appartement était occupé par des locataires. Le temps de libérer l'appartement et de le refaire à neuf, Patrizzia et son mari ont donc dû passer deux ans dans une résidence moderne de la proche banlieue avant de pouvoir revenir s'installer dans l'immeuble où Patrizzia avait passé son enfance.

\section{L'indivision prolongée du patrimoine, moteur des circulations}

Ce retard de l'installation dans les logements de famille se double d'un autre retard: celui du transfert de la propriété véritable des logements de famille aux enfants. En général, lorsque les parents «donnent» un logement de famille à l'un de leurs enfants, ils ne font que le mettre gratuitement à sa disposition, ou lui en donnent l'usufruit, mais ils en conservent généralement la propriété. Cette dernière reste concentrée dans les mains d'un des deux parents, puis entre celles du conjoint survivant à la mort de ce dernier, si bien que la propriété reste longtemps indivise et que c'est généralement quelques années avant la mort des parents qu'elle est finalement répartie entre leurs enfants, en venant confirmer - ou non - la répartition de la résidence déjà effectuée à l'occasion de leur mariage. Ce système de décalage chronologique entre répartition de la résidence et répartition de la propriété a l'avantage d'être très souple et de permettre d'adapter constamment le stock de logements de famille aux évolutions de la parentèle.

Le cas de la famille de Fabiana G. en fournit un bon exemple. Cette dernière est née à Naples en 1950 et descend en ligne paternelle comme en ligne maternelle de deux des plus vieilles familles nobles du royaume de Naples. Elle habite aujourd'hui dans le palais historique de sa famille maternelle, dans le quartier de Montedidio, dont le tiers est encore de propriété familiale, appartenant entièrement à la mère de Fabiana. Au moment du mariage de ses enfants, la mère de Fabiana leur a mis des logements à disposition dans le palais familial, mais en a conservé la propriété. Le frère aîné de Fabiana n'en a pas profité puisqu'il a émigré assez jeune à Milan et s'est établi dans le Nord. En revanche, Fabiana s'est mariée en 1974 après cinq ans passés dans un appartement en location situé à proximité dans le quartier. En attendant que l'appartement qui lui était destiné dans le palais familial se libère, elle est revenue s'installer dans le palais de Montedidio en 1979, d'abord dans l'appartement d'en face sur le même palier, puis dans son logement actuel. Dans les années 80, elle a en effet laissé son appartement à sa sœur qui, après quelques années passées à Padoue au lendemain de son mariage, est elle aussi revenue s'installer dans le palais familial lors de son retour à Naples. Sur incitation de sa mère, Fabiana lui a donc cédé l'appartement qu'elle occupait pour déménager sur le même palier. Ces échanges d'appartements entre frères et sœurs, ou les phénomènes de circulation d'un des enfants entre plusieurs logements de famille situés dans le même immeuble ou le même quartier sont assez fréquents et apparaissent comme le résultat d'un système de gestion familiale des logements fondé sur le maintien prolongé de l'indivision du patrimoine. 


\section{Mères et filles : arbitrages familiaux et circulations}

Ce système d'indivision prolongée du patrimoine place également les parents, et surtout les mères, en véritables chefs d'orchestre des mobilités familiales, ces dernières concentrant souvent l'essentiel de la propriété des logements de famille. Les inégalités de genre ont en effet continué à influencer fortement les pratiques successorales napolitaines jusqu'au moins à la mort des parents des personnes interrogées. Ainsi, dans les familles étudiées, les entreprises familiales et les maisons de famille rurales, symboles du prestige des lignées, étaient systématiquement transmises aux garçons et de préférence aux aînés, tandis que les logements napolitains étaient divisés de manière plus égalitaire, mais en privilégiant la proximité des filles. L'appartement parental, en particulier, était souvent divisé entre les filles, tandis que les garçons recevaient d'autres logements dans le même immeuble ou le même quartier. De même, lorsqu'un couple achetait un appartement, ce dernier était souvent mis au nom de la conjointe, le régime juridique de la séparation des biens étant largement dominant en Italie. Les entretiens offrent ainsi de nombreux exemples de femmes concentrant la propriété des logements familiaux et organisant les circulations à l'intérieur du système résidentiel (à l'image de la mère de Fabiana, citée ci-dessus). Les arbitrages qu'elles ont opérés entre leurs enfants ont reproduit largement les schémas traditionnels en privilégiant systématiquement la proximité des filles, ramenées prioritairement dans les immeubles familiaux, souvent sur le même palier ou à des étages voisins de ceux des parents, tandis que les garçons recevaient des logements de famille plus tardivement, ou se voyaient acheter des appartements en dehors de l'immeuble de famille. Cette importance de la proximité mère-fille se comprend du fait du rôle que les femmes jouent dans le soin des parents âgés en Italie, et en particulier à Naples, marquée par un très faible développement des services publics à la famille. Tout comme les puînés, qui ont souvent pu profiter d'une succession rapidement après leur décohabitation, les filles sont donc privilégiées par le système résidentiel patrimonial et leurs circulations sont moins intenses et plus circonscrites que celles des autres.

\section{Conclusion}

Cette étude permet de nuancer l'idée d'une déterritorialisation de la bourgeoisie contemporaine et montre la nécessité de dépasser, au sein de ce milieu social encore plus qu'ailleurs, l'opposition entre circulation et immobilité, sédentarité et mobilité, ancrage local et migrations internationales. L'émigration professionnelle et les circulations des élites napolitaines ont en effet largement contribué à maintenir leur ancrage local dans la ville, en permettant de libérer la pression sur le patrimoine familial et de faciliter le maintien des membres de la parentèle restés à Naples dans les quartiers les plus prestigieux de la ville. Dans ce milieu, circulation individuelle et immobilité familiale font système et se renforcent l'une l'autre, le départ des uns se révélant le plus souvent temporaire et permettant la réinstallation locale des autres et la reproduction de leur statut social.

Cette situation s'explique par le rôle clé du patrimoine familial, en particulier du patrimoine immobilier, dans la structuration des mobilités des élites italiennes. Mais elle est également liée au déclin de la bourgeoisie traditionnelle napolitaine, désormais prise dans une contradiction entre une injonction à la mobilité professionnelle d'une part, du fait de la crise économique profonde de la ville, et un prestige social encore largement fondé sur un patrimoine hérité et ancré localement, d'autre part. Dans un contexte d'amenuisement des fortunes, le «système résidentiel patrimonial» typique des élites napolitaines et italiennes, et fondé sur la réinstallation des enfants mariés dans des logements de famille prestigieux, a alors 
besoin de faire circuler les membres de la famille pour pouvoir fonctionner. L'association entre repli patrimonial local et noria migratoire apparaît comme une manière de gérer le déclin pour les vieilles familles de la bourgeoisie locale, qui s'installent durablement dans des territoires circulatoires entre Naples et les grandes métropoles européennes. On peut faire l'hypothèse qu'il s'agit là d'une attitude typique des élites patrimoniales de villes autrefois puissantes et devenues périphériques. D'autres travaux viendront approfondir ce rôle du déclin et du repli patrimonial dans les circulations de classes supérieures contemporaines, pour l'heure surtout étudiées dans des métropoles globales ou des centres majeurs de la mondialisation.

Thomas Pfirsch Maitre de conférences Université de Valenciennes UMR Géographie-cités thopfirsch@hotmail.com

\section{Bibliographie}

Allen, Judith ; Barlow, James ; Leal, Jesus et al. (2004) Housing and Welfare in Southern Europe, Oxford, Blackwell, 240 p.

Allum, Percy ; Allum, Marie Pierrette (1994) Naples telle qu'en elle-même, in Vallat, C. (dir) Naples. Le paradis et les diables, Paris, Autrement, pp. 104-118.

Barbagli, Marzio ; Castiglioni, Maria ; Dalla Zuanna, Gianpiero (2003) Fare famiglia in Italia, Bologne, Il Mulino, 308 p.

Beaverstock, Jonathan V. (2001) Transnational elite communities in global cities : connectivities, flows and networks, $G A W C$ research bulletin, $\mathrm{n}^{\circ} 63$ [en ligne] URL : $<$ http://www.lboro.ac.uk/gawc/rb/rb63.ht $\underline{\mathrm{ml}}>$.

Bertaux-Wiame, Isabelle (1991) La force de rappel des liens familiaux. Rapports intergénérationnels et trajectoires familiales, in Bawin Legros, B.; Kellerhals, J. (dir) Relations intergénérationnelles, Genève, Université de Liège, pp. 185-196.

Bonvalet, Catherine; Maison, Dominique (1999) Famille et entourage: le jeu des proximités, in Bonvalet, C. ; Gotman, A.; Grafmeyer, Y. (dir.) La famille et ses proches. L'aménagement des territoires, Paris, PUF, pp. 27-69.

Brancaccio, Luciano; Zaccaria, Anna Maria (2003) La classe dirigente napoletana. Caratteri, risorse e configurazioni relazionali, in Amaturo, E. (ed.) Capitale sociale e classi dirigenti a Napoli, Rome, Carocci, pp. 115154.

Duclos, Denis (2002) Société-monde. Le temps des ruptures, Paris, La découverte, 256 p.

Dureau, Françoise (2002) Les systèmes résidentiels: concepts et applications, in Lévy, J-P ; Dureau, F. (dir.) L'accès à la ville. Les mobilités spatiales en questions, Paris, L'Harmattan, pp. 355-382.

Finch, Janet (1989) Kinship and Friendship, In Jowell, R. ; Witherspoon, S. ; Brook, L. (eds.) British Social Attitudes. Special International Report, Aldershot, Gower, pp. 87-104. 
Firth, Raymond ; Hubert, Jane ; Forge, Anthony (1969) Families and their Relatives. Kinship in a middle-class sector of London: an anthropological study, London ; New York, Routledge \& Kegan Paul ; Humanities press, $492 \mathrm{p}$.

Hoffmann-Nowotny, Hans-Joachim (1970) Migration. Ein Beitrag zu einer soziologischen Erklärung, Stuttgart, Enke, 155 p.

Höllinger, Franz; Haller, Max (1990) Kinship and social networks in modern societies: a cross-cultural comparison, European Sociological Review, nº, pp. 103-124.

Le Bris, Emile; Marie, Alain; Osmont, Annick; Sinou, Alain (1987) Famille et résidence dans les villes africaines, Paris, L'Harmattan, 220 p.

Martellini, Amoreno (1997) Fare il milione : la emigración de élite y el mito de la tierra prometida, Estudios Migratorios Latinoamericanos, vol. 12, n³7, pp. 467-489.

Pfirsch, Thomas (2008) Des territoires familiaux dans la ville. Classes supérieures, relations familiales et espace urbain à Naples, Paris, Université Paris Ouest Nanterre, 432 p. Th. Doct: Géogr: Paris Ouest Nanterre: 2008

[en ligne] URL: http://tel.archivesouvertes.fr/tel-00683824

Pfirsch, Thomas (2011) Une géographie de la famille en Europe du Sud, Cybergeo: European Journal of Geography, article 533 [mis en ligne le 25 mai 2011] URL: $<$ http://cybergeo.revues.org/23669>.

Pierre, Philippe (2005) Les variations de l'identité d'élites mondialisées, Connexions, n83, pp. 191-210.

Pinçon, Michel ; Pinçon-Charlot, Monique (1989) Dans les beaux quartiers, Paris, Seuil, $255 \mathrm{p}$.

Rosental, Paul-André (1999) Les sentiers invisibles. Espace, familles et migrations dans la France du 19è siècle, Paris, Ehess, 255 p.

Wagner, Anne Catherine (1998) Les nouvelles élites de la mondialisation. Une immigration dorée en France, Paris, PUF, 240 p.

Wagner, Anne Catherine (2003) La bourgeoisie face à la mondialisation, Mouvements, n²6, pp. 33-39. 